\title{
ETSI DCC: Decentralized Congestion Control in C-ITS
}

\author{
Nikita Lyamin*, Alexey Vinel*, Dieter Smely ${ }^{\dagger}$, Boris Bellalta ${ }^{\ddagger}$ \\ *Halmstad University, Sweden. name.surname@hh.se \\ ${ }^{\dagger}$ Kapsch TrafficCom AG, Austria. dieter.smely@kapsch.net \\ ${ }_{\ddagger}^{\ddagger}$ Universitat Pompeu Fabra, Spain. boris.bellalta@upf.edu
}

\begin{abstract}
Decentralized Congestion Control (DCC) is a mandatory component of 5.9 GHz Intelligent Transportation Systems (ITS-G5) vehicular communication protocol stack that reduces radio channel overload, range degradation, and self interference. In this tutorial article we explain its principle, describe related ongoing standardization activities, evaluate its performance for emerging cooperative driving applications, and identify ways for improvement. We show that failure to use a proper DCC parameterization can impact negatively on the performance of cooperative vehicular applications.
\end{abstract}

\section{INTRODUCTION}

Cooperative Intelligent Transportation Systems (C-ITS) use the connectivity between vehicles, roadside infrastructure, and other road users to enhance driving safety and comfort, and improve traffic management. Regular exchange of information between road users (beaconing) keeps them informed about each other's position, driving kinematics, and other attributes. This is one of the cornerstone of road safety and traffic efficiency applications on the way towards autonomous driving.

To facilitate C-ITS, the European Telecommunication Standards Institute (ETSI) in ETSI EN 302665 specified the ITS-G5 architecture and a communication protocol stack to be used in the $5.9 \mathrm{GHz}$ spectrum. ITS-G5 adopts the medium access control (MAC) and the physical (PHY) layer techniques from the IEEE 802.11 standard like the widely adopted Wi-Fi.

C-ITS communications must also be operational in dense road traffic. Assuming that all vehicles participate in the CITS information exchange by broadcasting periodic messages, wireless channel congestion is likely to occur. Thus, to avoid degradation of the system performance caused by too high a channel load and provide a fair access to the channel resources among neighboring ITS-G5 stations (ITS-S), channel congestion control mechanisms are required. To this end ETSI published TS 102687 [1] - a specification of a decentralized congestion control (DCC) mechanism - as a part of the ITS-G5 protocol stack. DCC is a mandatory component of ITS-S operating in the $5.9 \mathrm{GHz}$ frequency band.

However, restricting the communication exchange in safety-critical applications, which are very delay-sensitive could potentially lead to undesirable performance degradation. Several studies show, that the performance of the ETSI DCC needs further investigation [2], to find more efficient DCC parameter settings than the default one, to prevent possible performance degradation of C-ITS [3]. Thus, in this tutorial paper we first present an overview of the state-of-the-art in DCC for vehicular communications. Then we evaluate the performance of the cooperative driving application enabled by ETSI ITS-G5 communications via several simulation experiments to assess the potential influence of DCC on the performance of the system.

The paper is organized as follows: Section II provides a tutorial on the standardization and the related research literature. In Section III we describe the system model, including the principal assumptions on the cooperative driving scenario considered, the mobility model and the communication setups. In Section IV we present the simulation study of C-ITS system enabled by ITS-G5 vehicular communication stack, discuss the results, and give recommendations for further development. Finally, Section V contains the conclusions drawn from the study.

\section{STANDARDS AND LiteratURE}

\section{A. ETSI DCC standardization}

ETSI is an international standardization body based in France. ETSI develops standards - European Norms (EN) and Technical Specifications (TS) - comprising normative requirements that enable interoperability between the devices of different vendors and also avoid harmful radio interference. ETSI also develops non normative documents that contain additional information or guidance. The drafting of the documents is done by Technical Committees (TC), consisting of delegates of ETSI members and by expert groups called Specialist Task Force (STF) consisting of selected experts. All C-ITS documents are drafted by the TC ITS and the associated five Work Groups (WG1 to WG5) temporarily supported by STFs.

ETSI TC ITS WG4 first introduced DCC in ETSI TS 102687 [1] and this standard is the focus of our work. It introduced a state machine approach at access layer to adapt several transmission parameters to the measured channel load. Each state is associated with a certain channel load range and a set of transmission parameters. Our study presented in this article inspired the revision of [1]. In this revision [4] the DCC algorithms are adapted to the channel load limit approach specified by ETSI TC ITS WG2 in ETSI TS 103175 [5]. Specification [4] also allows for different algorithms to be implemented. 
DCC can operate as gatekeeper on the medium access layer, but higher layer DCC functionalities are possible, as specified in [5]. DCC as specified in [1] is based on a state machine that has three states: Relax, Active and Restrictive, as shown in Figure 1.a.

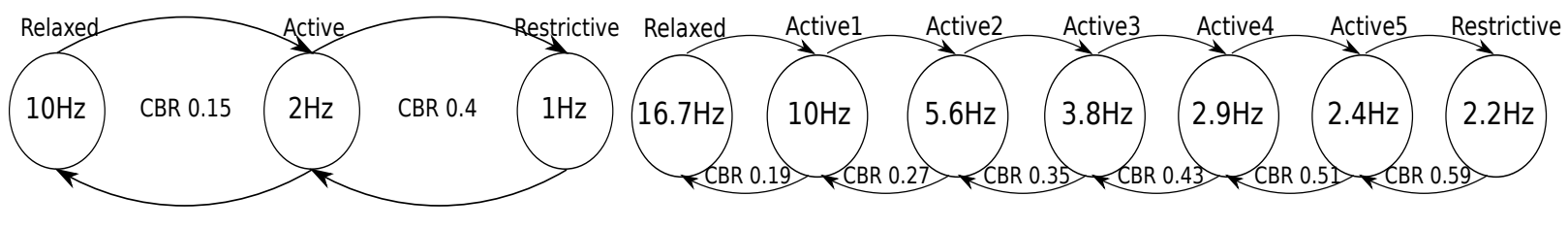

a) ETSI TS 102687 Configuration, "DCC 2+1"

c) ETSI TR 101612 Configuration, "DCC 2+5"

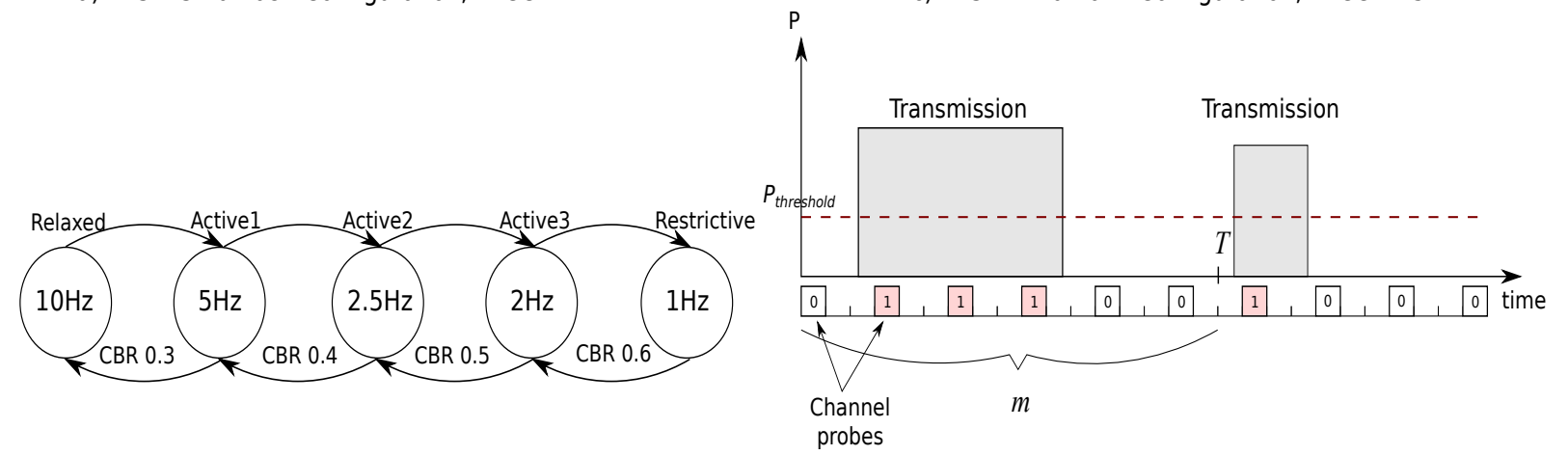

b)ETSI TS 103175 Configuration, "DCC 2+3"

d)Channel Busy Ratio (CBR) measurement

Fig. 1: DCC state machines and $C B R$ measurement procedure ( $m$ is the number of channel probes within the measurement period $T$ )

In each DCC state the restrictions on the transmission parameters are defined. ETSI DCC in general considers the five following mechanisms to control the vehicle's channel access: "Transmit Power Control" (TPC), "Transmit Rate Control" (TRC), "Transmit Datarate Control" (TDC), "DCC Sensitivity Control" (DSC), "Transmit Access Control" (TAC).

The choice of the DCC state is performed based on the evaluation of a so-called Channel Busy Ratio (CBR). ETSI suggests the following reference method to estimate the value of $C B R$. The ITS-S makes periodic channel probes and calculates the proportion of time the channel was busy during a measuring interval $T=1 s$ [1]. To calculate the time the channel was occupied, the ITS-S should take $m$ measurements of the received signal level uniformly distributed within the measuring interval. The time between two channel probes should be set to detect the transmission of the smallest possible packet at the highest available datarate. For all channel probes of length $10 \mu$ s the average signal level $P$ is determined. Then the $C B R$ measure for the received signal level threshold $P_{-}$threshold $\left(-85 \mathrm{dBm}\right.$ by default) is given as: $C B R=\sum_{i=1}^{m}$ (probes with $P>P_{-}$threshold) $/ \mathrm{m}$, as shown in Figure 1.d.

Each transition in the state machine has a corresponding $C B R$ value as threshold (Fig. 1), the transition is performed under one of the two following conditions:

1) Transition to a more restrictive state: If the $C B R$ value was above the threshold during the last observed measuring interval.

2) Transition to a less restrictive state: If the $C B R$ was below the threshold during the last five consecutive observed measuring intervals.

In each state of the state machine, TRC specifies the minimum time interval between two subsequent transmissions, i.e. TRC specifies the maximum possible transmission rate (messages per second) for each appropriate state.

ETSI DCC also allows state-machine configurations containing a set of sub-states in the "Active" state. This approach enables finer granularity of the DCC state transitions possible. The state machine is fully meshed to allow for transitions between any two states, depending solely on the $C B R$ measurements history, i.e. in defining the current state DCC relies only on the recent CBR measurements and may switch from one state to another in a single step. For simplicity, transitions between non-neighboring states are omitted in Figure 1. Thus, DCC-configurations with a reasonable number of sub-states may help prevent rapid changes in the C-ITS transmission behavior, maintaining the targeted level of congestion. Figures 1.b and 1.c show the implementations of the DCC state machine with additional number of sub-states in Active state, taken from [5] and [6], accordingly.

Hereafter, we referred to 3 DCC configurations shown in Figure 1 as "DCC 2+1", "DCC 2+5", "DCC 2+3" by the number of sub-states in the Active state of the DCC state machine. 


\section{B. Literature overview}

The performance of ETSI DCC has been discussed in several studies. The authors of [2] present an extensive performance evaluation of the 3-states ETSI DCC for various CBR values. Based on simulation results, the paper considers the effectiveness of various ETSI DCC CBR control mechanisms (TPC, DSC, TRC, DCC) from the communication and application point of view. Other studies of ETSI DCC demonstrated that the basic 3-state DCC configuration may show low performance. In [7] it is demonstrated that the basic 3-state configuration of DCC [1] tends to oscillate, i.e. to repeatedly switch between relaxed to active and restrictive states. The "unfairness" of the 3-state ETSI DCC configuration [1] was also explained in [3]. The authors show that in a high vehicle density scenario ITS-S may experience unfairness in terms of channel access. The reason for this is a condition when two neighboring ITS-S (i.e. that are in the same communication range) choose different states of the DCC state-machine. Based on the simulation study presented in [8], the authors conclude that a DCC state machine with 3 states has poor performance in terms of its ability to adjust its state to varying CBR values.

Thus, alternative ETSI DCC configurations and parameter sets have been proposed in the literature to overcome aforementioned drawbacks. For example, in [9] the focus is on to the tuning of the TDC configuration. Following the outcome of their previous study, in [10], the authors propose using only TDC (transmit datarate control) for a 3-state DCC configuration, keeping the transmit power level and the sensitivity level for all states at a constant value equal to the Active state of the ETSI DCC 3-state configuration of [1]. In [9] the authors also focus on adjusting the TDC. The novelty of their DCC design is that the switch between different DCC states is performed using a hysteresis curve for the CBR instead of conventional thresholds. The hysteresis mechanism allows a better control of the CBR trend based on the last measurement interval. It also allows different CBR values for the same state, depending on whether the local CBR increases or decreases in comparison to the previous measurement interval.

Another way to enhance DCC performance suggested in the literature is to increase the number of sub-states in the active state. Thus, the authors in [8] propose a 6-state DCC configuration based on TPC in combination with different CBR thresholds for each state to introduce a negative feedback to the control loop. To obtain the CBR thresholds, the authors identify the channel load that they considered to be an optimum balance between improving channel utilization and packet collisions and select the state transition parameters so that the state machine operates close to this optimal channel load. The target CBR value was identified through simulations of various vehicular densities. The simulation results presented in the paper show that a CBR value of 0.65 is a reasonable value for the channel load, regardless of the vehicle density or the CBR threshold.

Following a similar approach, the authors in [11] propose the use of DCC with several sub-states in Active state together with TPC. The CBR thresholds for the state transitions are selected according to CCA (clear channel assessment) value. The authors introduce a TRC implementation that gradually decreases the beaconing rate from $10 \mathrm{~Hz}$ to $1 \mathrm{~Hz}$ following the increase of CBR. Finally, it was shown that a DCC configuration with more Active sub-states has a better performance due to its improved adaptivity to varying CBR values.

Other attempts are taken in the direction of avoiding the ETSI DCC re-active state-machine involve controlling the CBR pro-actively. In [12] the authors perform a simulation study to compare the performance of the ETSI DCC state machine with several sub-states in Active state with a linear adaptive DCC packet rate control mechanism called LIMERIC. For both configurations, only TRC is considered. In the presented setup LIMERIC outperforms the state-machine approach in terms of IPG (inter packet gap).

\section{PRerequisites}

\section{A. Scenario}

The simulation study presented in this paper focuses on the C-ITS use case named platooning [13], which is one of the applications considered to be an early adopter of C-ITS technology. In platooning the leading vehicle is driven by a professional driver, while the following vehicles execute at least longitudinal automatic control or can even be switched to a fully autonomous mode with a lateral control, as well. The main purpose of platooning is to reduce air-drag in a caravan of heavy-duty vehicles, which can significantly improve fuel consumption.

We choose platooning as an illustrative C-ITS application example to show how even small variations in the configuration of ITS-G5 communications may affect the performance of safety and time-critical C-ITS applications. At present, prestandardization activities are still ongoing at ETSI TR 103301 and TR 103 299, which are studying the applicability of currently available standards for platooning applications [13]. Therefore, a way to enable functioning of the platoon's automatic control system based on the current standardization framework is considered in this article (ETSI specifications [1], [5], and [6]), which could be summarized as follows:

- A vehicle generates Cooperative Awareness Messages (CAMs) based on one of the following approaches:

- fixed triggering frequency $f_{\text {CAM }}$ messages per second (henceforth called, static CAM).

- kinematic-based triggering in accordance with ETSI EN 302 637-2 (henceforth called, ETSI CAM). In ETSI CAM a vehicle generates new CAMs depending on its current speed, acceleration, deceleration, and change of direction [14].

- The time elapsed since last CAM transmission is checked for compliance with TRC. In the case the TRC timer is still active, the CAM is queued until the TRC timer elapses, or dropped when the CAM lifetime expires. 
- If the CAM is not dropped, it is transmitted on the dedicated channel in accordance with the CSMA/CA (Carrier Sense Multiple Access / Collision Avoidance) 802.11p MAC protocol. Currently ETSI allocates five channels of $10 \mathrm{MHz}$ bandwidth each (one management channel and four service channels). We assume that platooning operates in one of the four dedicated service channels.

In order to test the effect of various legacy communication configurations on the performance of the platooning application we assume 12 setups, that are obtained by combining of 3 DCC configurations ("DCC $2+1$ ", "DCC 2+3", "DCC 2+5") [1], [5], [6], and 4 CAM generation policies (three static CAM: $10 \mathrm{~Hz}, 20 \mathrm{~Hz}, 30 \mathrm{~Hz}$, and ETSI CAM).

In this article we focus on DCC based on TRC, i.e. a state machine for the packet rate control. A pure packet rate control mechanism has several advantages: most importantly it is easy to implement and has an immediate impact on the channel load across the entire radio range. Other concepts, also described in ETSI TS 102 687, either need information exchange between the ITS-S to control the local $C B R$ (datarate control) or influence the communication range (transmit power control). The latter has disadvantages for platooning, since envisaged control strategies require that all the vehicles in the platoon are within the same communication range, enabling one-hop connectivity from the leader to all members. In addition, there are no DCC parameters for TPC, TDC, DSC and TAC available in the literature ( [5], [6]) for the configurations shown in Figures 1.b and 1.c.

To test the performance of DCC and both kinematic-based and fixed beaconing approaches, we study the following reference scenario:

- We consider a platoon consisting of $N$ vehicles moving along a highway.

- The platoon moves along the straight stretch of a road with a target speed for the leading vehicle $V$, and a target gap $d$ between platoon members. Each vehicle in the platoon adapts its speed according to the kinematic information received from the platoon leader and the preceding vehicle. We use a longitudinal control algorithm based on the sliding surface method of the controller design.

- For the speed pattern we assume a "disturbance scenario" as introduced in [14] (see Figure 2). The scenario reflects the situation when a slower vehicle approaches the right-most lane from a highway ramp or after a lane change.

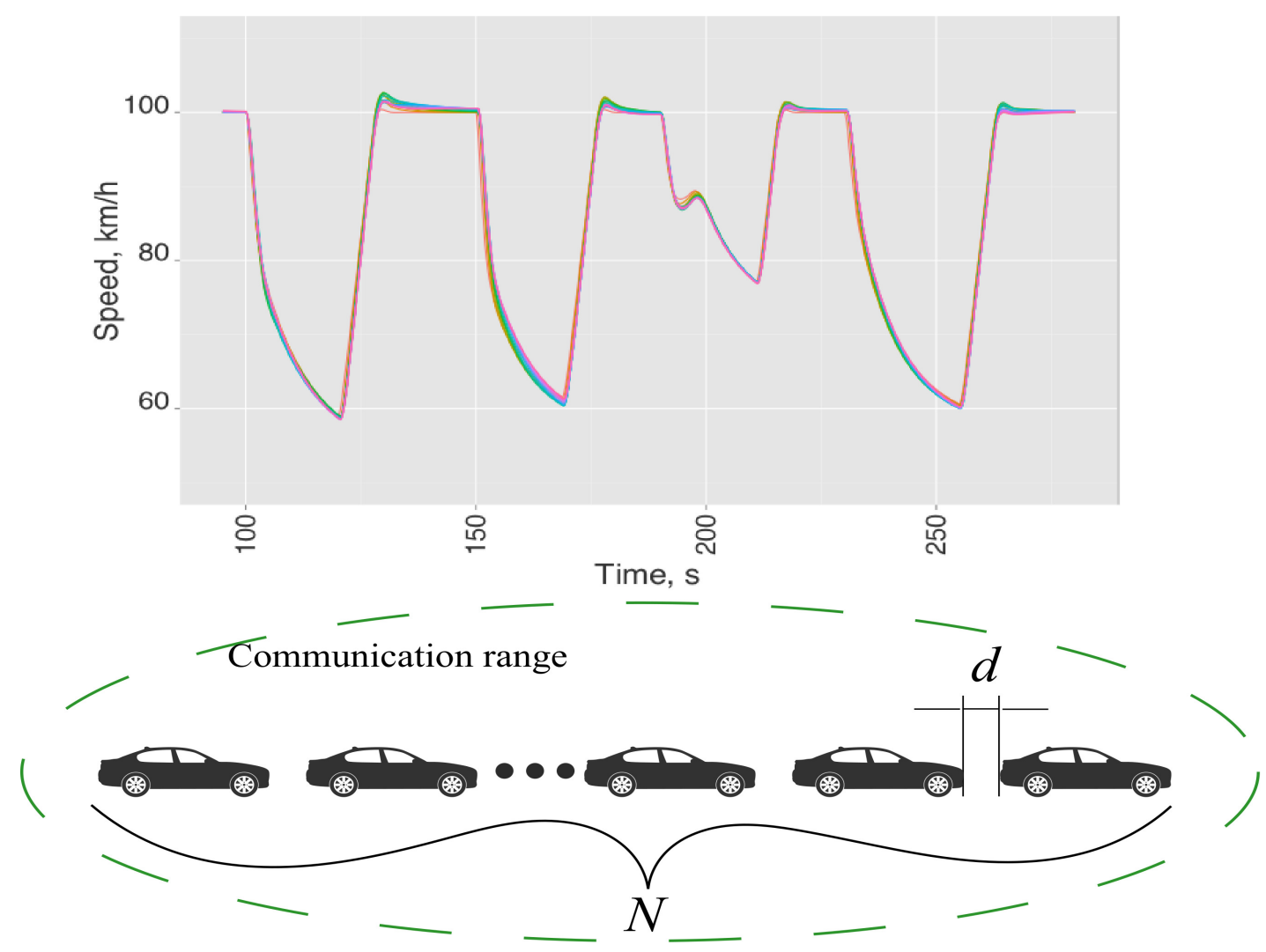

Fig. 2: Reference scenario: color lines represent example speed profiles of the vehicles

\section{B. Performance metrics}

We use the following metrics for performance evaluation: 
1) Box-plot of inter-vehicle gaps. The variation of inter-vehicle gaps reflects the ability of platoon members to maintain the target distance from the preceding vehicles. In [15] it is shown that the ability of a platoon to keep precise inter-vehicle gaps contributes to its fuel efficiency. We draw box plots of inter-vehicle gap distances for all vehicles in the platoon and for all runs of a given scenario. In each box, the central red mark indicates the median, and the bottom and top blue edges of the box indicate the $25^{\text {th }}$ and $75^{\text {th }}$ percentiles, respectively. The whiskers extend to the most extreme data points not considered as outliers, and the outliers (minimum and maximum values in a data set) are plotted individually using the red '+' symbol.

2) Box-plot of the $C B R$. The channel busy ratio characterizes the performance of DCC and its ability to keep the channel load below congestion. As with the inter-vehicle gap, we present $C B R$ measurements in the form of a box plot.

3) Probability Mass Function (PMF) of the data age $(f(t))$. The data age is a random variable defined as the time elapsed since the last successfully received packet of vehicle $2 \leq n \leq N$ by the leading vehicle. Data age is the difference between the current point in time and the latest point when the leading vehicle has successfully received a CAM from a vehicle $n$ [13]. In this article, the PMF of the data age for the last vehicle $N$ in the platoon is calculated, in order to study the farthest pair of communicating nodes.

\section{Performance eVAluation}

\section{A. Simulation environment}

In our simulation study we use Plexe - a simulator that supports a realistic simulation of a platooning system. The simulator is a combination of the two well-known and widely-used simulators Omnet++ and SUMO. Omnet++ is an event-driven network simulator that models the network part, while SUMO handles the mobility of the nodes (vehicles).

Our simulation setup uses the following parameters: The platoon consists of $N=15$ vehicles with a target inter-vehicle gap $d=5 \mathrm{~m}$, and a platoon leader target speed of $V=100 \mathrm{~km} / \mathrm{h}$. We also implemented the DCC state machine together with the kinematic-based ETSI CAM triggering mechanism, to meet the standardization requirements. Static CAM triggering policies with $F_{C A M}=\{10,20,30\} \mathrm{Hz}$ and kinematic-based ETSI CAM triggering with a sampling rate $T$ _CheckCamGen $=50 \mathrm{~ms}$ are tested. The size of the CAM message is $L=2000$ bytes at a data rate of $R=6 \mathrm{Mbit} / \mathrm{s}$.

We set the transmission power level to $23 \mathrm{dBm}(200 \mathrm{~mW})$ EIRP, which is the maximum allowed value that can be used in SCH1. The radio channel is simulated by a log-distance path loss model with a path loss exponent $\gamma=2$. For the reference length of a vehicle - 5 meters - this setup guarantees that even the last platoon member can detect unequivocally an ongoing transmission or packet collision.

The $C B R$ estimation conforms to the standardized procedure [1] as described in Section II, but for the simplicity of implementation it is adjusted to our modeling assumptions in the simulator. Since in our setup the duration of all CAM transmission times is the same $(L / R)$, and all the vehicles are in each other's communication range, an ITS-S counts the number of message transmissions $M$ observed (including CAM collisions) during $T$ and applies the formula $C B R=(M \cdot L / R) / T$.

\section{B. Discussion}

Figure 3 shows the box plot for the inter-vehicle distances and Figure 4 shows the box plot of $C B R$ for all the considered communication setups. The plots are clustered in 3 groups based on the number of DCC states.

In all setups, the average inter-vehicle gap is kept close to the desired $d$ of 5 meters. It would be logical to assume, the higher the CAM rate, the better the performance of the platoon, which however, is not always the case. It is noticeable, that for all three DCC configurations ETSI CAM outperforms the static CAM beaconing approach ( see Figure 3). This is because ETSI CAM triggers more messages when the ITS-S behavior is highly dynamic and fewer messages for the constant speed movement. Thus, the $C B R$ generated by the ITS-S mimics the platoon's speed pattern (see Figure 5). In other words, the channel is not overloaded when the platoon maintains a stable speed, while channel occupancy grows whenever maneuvers are performed. Such patterns give time for the vehicles to exchange more messages before DCC starts to react on the $C B R$ increase, which at the same time comes at the expense of higher $C B R$ peak values (see Figure 4).

According to [6], when the number of vehicles in the communication range is below 100, DCC should be able to maintain the $C B R$ level below 0.55. In our setup the $C B R$ value never exceeds higher than 0.4, although without DCC the CAM messages transmitted at $F_{C A M}=30 \mathrm{~Hz}$ may easily overload the channel (the rough estimation of the traffic $N$ vehicles may create without DCC is $\frac{N \cdot F_{C A M} \cdot L}{R} \approx 1.2$ ). From this we conclude that all three configurations of DCC can control $C B R$ at an allowable level.

Configuration "DCC 2+1" demonstrates inferior performance to the other two configurations, due to limiting the transmission rate even the $C B R$ is well below 0.55 (see Figure 4). This is due to an absence of intermediate sub-states in the "active" state: Whenever the state machine makes a transition from the "relaxed" to the "active" state, it has a $2 \mathrm{~Hz}$ allowed transmission rate, while "DCC $2+3$ " and "DCC $2+5$ " have a better granularity in terms of allowed transmission rate values (see Figure 1).

Figure 6 shows the data-age PMF distributions for all three DCC setups with the ETSI CAM. For "DCC 2+1" most of the data-age values are around $0.1 \mathrm{~s}$ and $0.5 \mathrm{~s}$, which corresponds to "Relaxed" $(10 \mathrm{~Hz})$ and "Active" $(2 \mathrm{~Hz})$ states. The reason for significant density values around $0.15 \mathrm{~s}$ when using the ETSI CAM is as follows. When the platoon maintains a speed of 


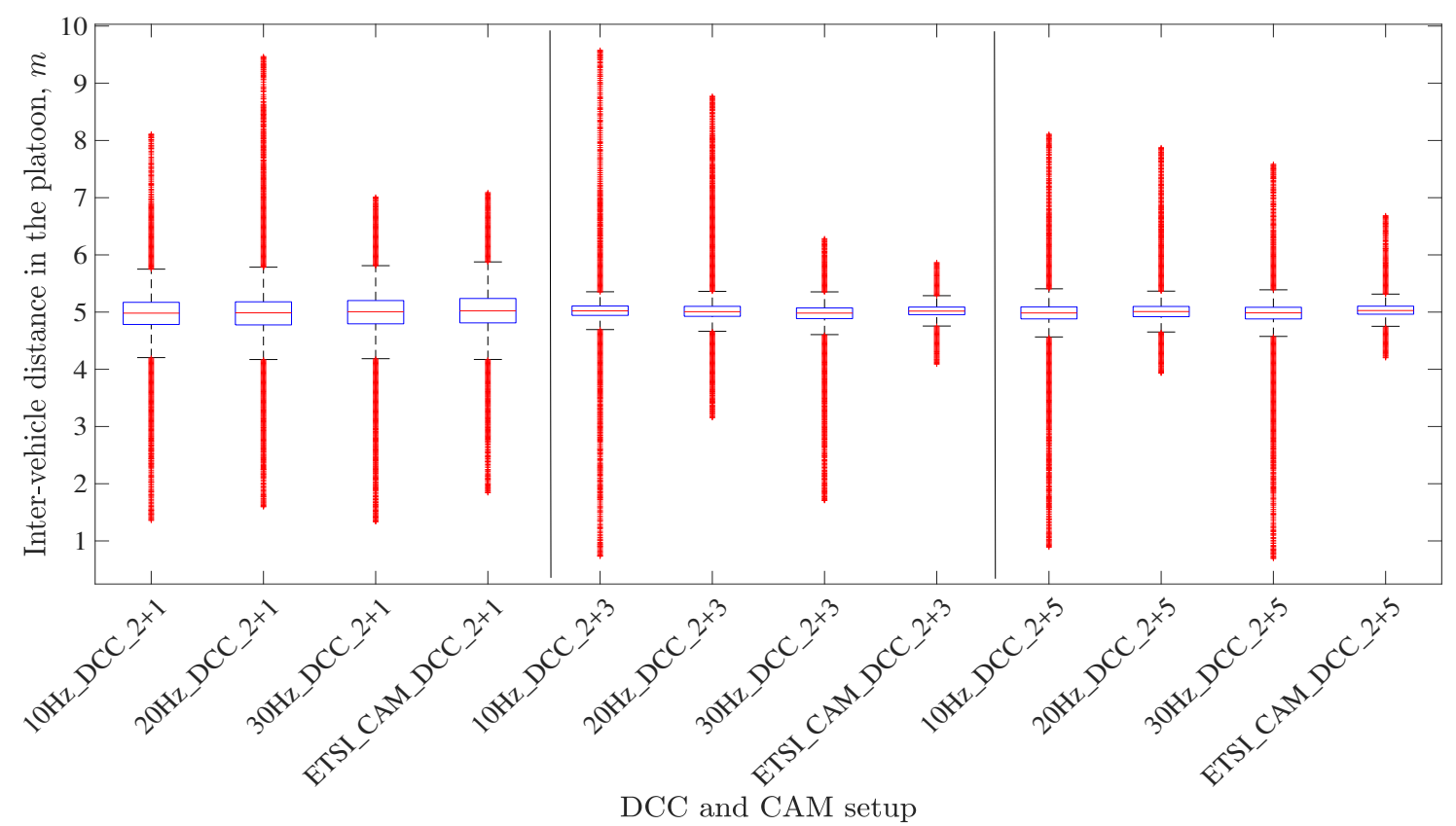

Fig. 3: Inter-vehicle distances

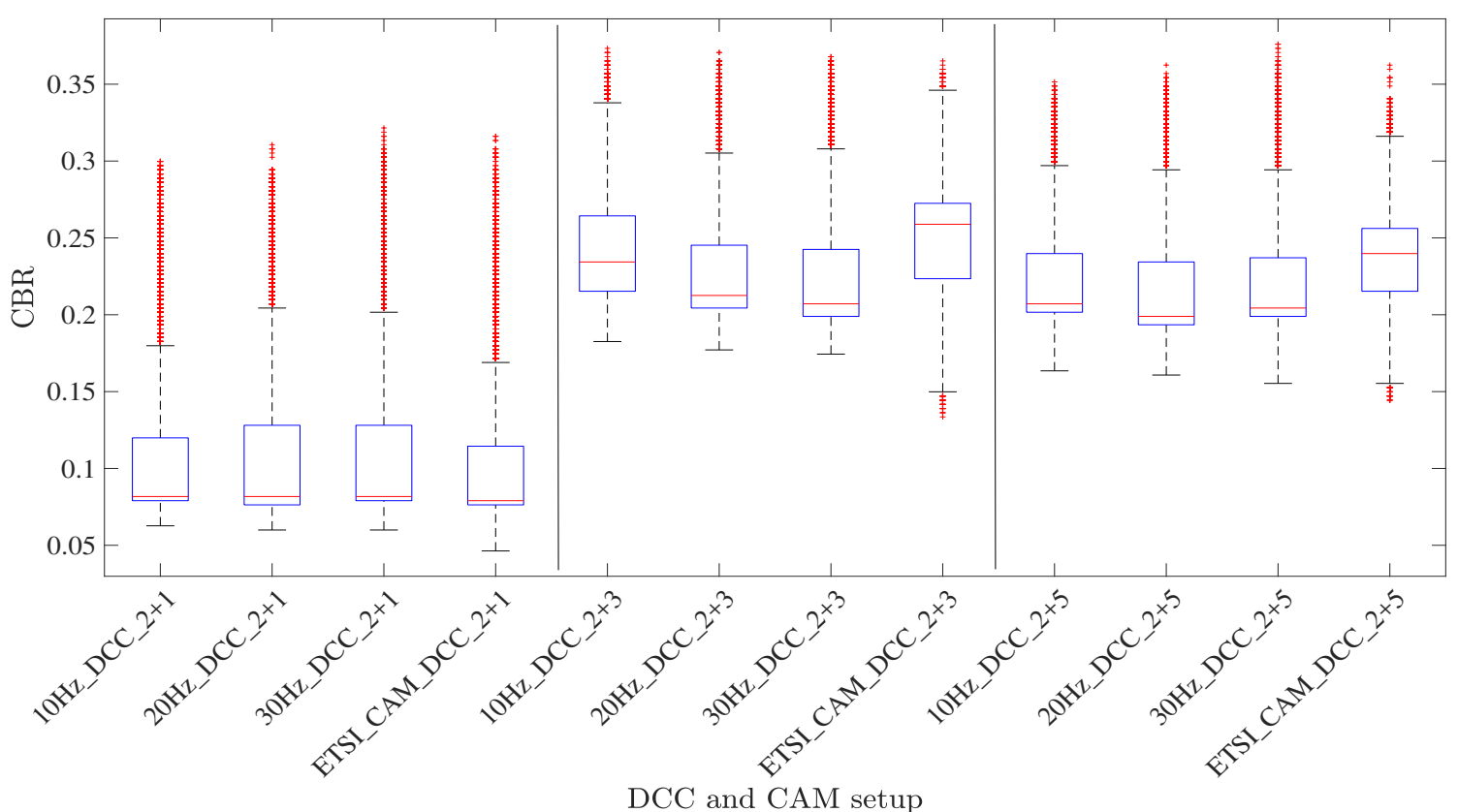

Fig. 4: Channel Busy Ratio

$V=100 \mathrm{~km} / \mathrm{h}(27.8 \mathrm{~m} / \mathrm{s})$ the CAMs are triggered with $\frac{27.8}{4} \approx 7 \mathrm{~Hz}$, which corresponds to a data-age value of $1 / 7 \approx 0.15 \mathrm{~s}$. Similarly, for "DCC $2+3$ " and "DCC $2+5$ " the PDF maximum values are concentrated around the rates defined by the TRC configuration of the corresponding DCC state machine. Thus, we conclude that the configuration of the DCC state machine has a direct impact on the data age distribution shape. It can be seen that the data age values are grouped exactly at the points specified by the DCC TRC configuration and almost never take any other values. This fact provides us with empirical evidence, that platooning may benefit from DCC configurations with more states, which could allow smoother control of data age while maintaining the required $C B R$ level. 

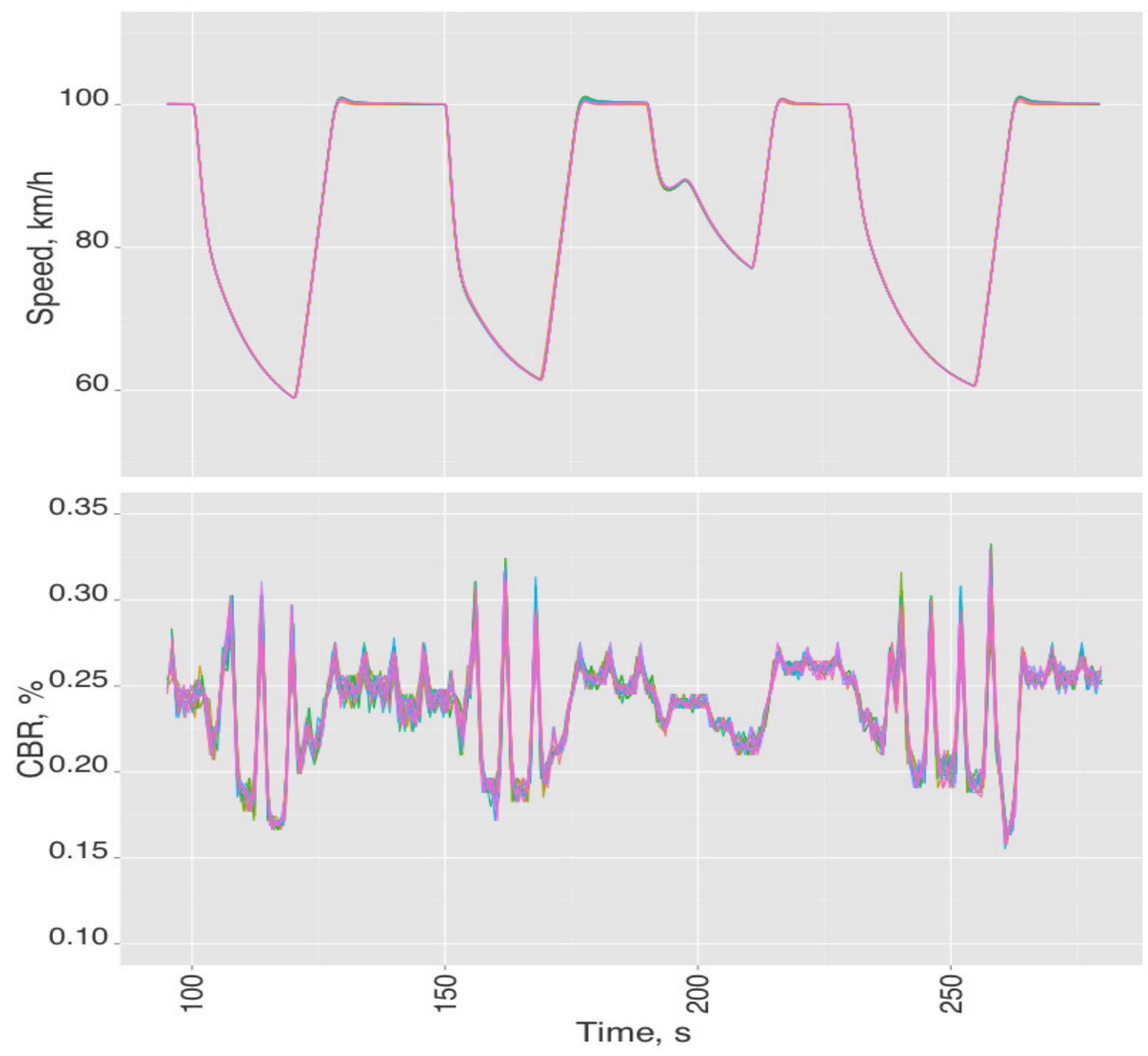

Fig. 5: Representation of $C B R$ measurements for one experiment run using "DCC 2+5 ETSI CAM": color lines represent different platoon members.

\section{CONCLUSiON AND OPEN CHALlEngeS}

ETSI standardization on the Decentralized Channel Control (DCC), which is a crucial element in controlling the channel load in ITS-G5-based C-ITS networks, is ongoing. Currently, according to ETSI, a satisfactory range for the channel busy ratio $(C B R)$ is 0.55 to 0.75 [6]. From our study, we conclude that for the legacy ETSI DCC configurations, when the number of closely located cooperatively driving vehicles is below 15 , the $C B R$ value is always less than 0.4 , even if the assumed message length is as great as 2000 Bytes. We demonstrate that the unnecessary low values of the actual $C B R$ generated by the current ETSI DCC configurations, will have a major negative impact on the performance of C-ITS applications in terms of achieved data age. The under-utilized channel resources for time-critical applications demonstrate the need for further DCC optimization, which includes:

- A justified approach for the selection of $C B R$ thresholds for the ETSI DCC state-machine configurations or a more efficient DCC mechanism/algorithm is required.

- To date, the parameter values settings for ETSI DCC CBR control mechanism, e.g. TPC, TDC and DSC are not well specified in the existing ETSI specifications and need further development.

Having said the above, the most importantly, we would emphasize that the currently specified ETSI DCC configurations are designed to control the $C B R$ level as such, but not the system level C-ITS application metrics. Standards for several safety-critical C-ITS applications (e.g. platooning) are currently under development. Appropriate control criteria for channel congestion level could be selected to make the DCC to target at optimizing the applications performance metrics. For this purpose, mechanisms are needed to estimate the influence of the $C B R$ limits on the performance of C-ITS applications. 


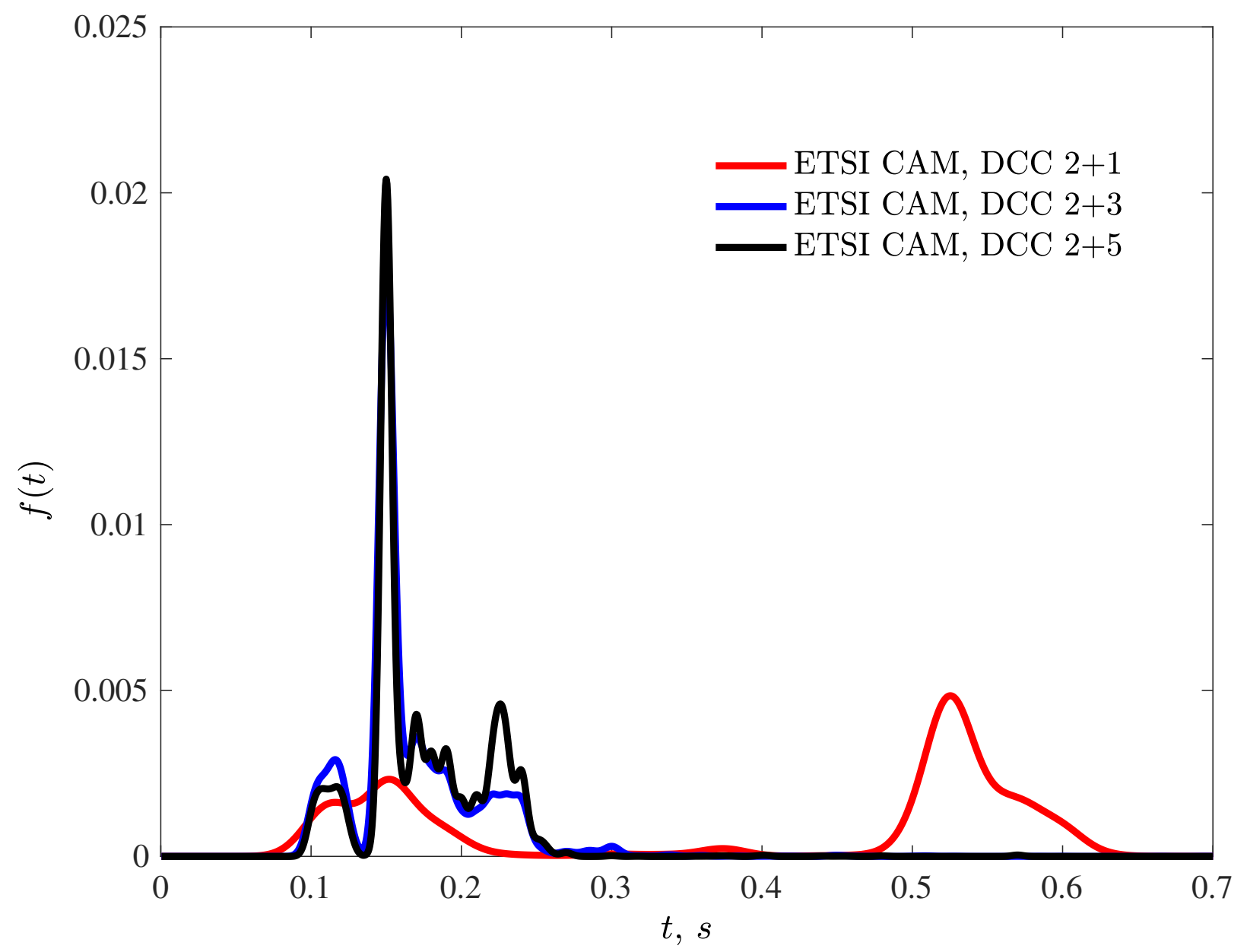

Fig. 6: Empirical probability density function of the data age

In our opinion, mathematical models of the DCC are clearly needed to better characterize and understand the complex dynamics of C-ITS systems further. This demand is especially emerging, since the studies of the ETSI DCC which are currently available in the literature [8]-[11] rely on the simulation experiments of specific scenarios and the theoretical foundations to develop the DCC configurations are required.

The results presented in this article were used as one of the inputs for revising [1]. A new version of the ETSI DCC standard [4] includes the following modifications:

- states in state machine are no longer meshed and are adapted to [5],

- TAC and DCS were removed,

- a restriction on the transmission duration was introduced,

- the measurement period $T$ was reduced,

- an alternative linear control algorithm was introduced.

However, the open questions presented above have not yet been fully addressed yet.

\section{ACKNOWLEDGMENTS}

The research leading to the results reported in this work has received funding from the Knowledge Foundation (Sweden) and from the ELLIIT Strategic Research Network.

\section{REFERENCES}

[1] "Intelligent transport systems (ITS); decentralized congestion control mechanisms for intelligent transport systems operating in the 5 GHz range; access layer part," ETSI TS 102687 V1.1.1, 2011.

[2] A. Autolitano, C. Campolo, A. Molinaro, R. M. Scopigno, and A. Vesco, "An insight into decentralized congestion control techniques for VANETs from ETSI TS 102687 V1. 1.1,” in 2013 IFIP Wireless Days (WD), pp. 1-6, November 2013. 
[3] S. Kuk and H. Kim, "Preventing unfairness in the ETSI distributed congestion control," IEEE Communications Letters, vol. 18, pp. 1222-1225, July 2014.

[4] "Intelligent transport systems (ITS); decentralized congestion control mechanisms for intelligent transport systems operating in the 5 GHz range; access layer part," ETSI TS 102687 V1.2.1, 2018.

[5] "Intelligent transport systems (ITS); cross layer DCC management entity for operation in the ITS G5A and ITS G5B medium.," ETSI TS 103 175 V1.1.1, 2015.

[6] "Intelligent transport systems (ITS); cross layer DCC management entity for operation in the ITS G5A and ITS G5B medium; report on cross layer DCC algorithms and performance evaluation," ETSI TR 101612 V1.1.1, 2014.

[7] D. Eckhoff, N. Sofra, and R. German, "A performance study of cooperative awareness in ETSI ITS G5 and IEEE WAVE," in 2013 10th Annual Conference on Wireless On-Demand Network Systems and Services, WONS 2013, pp. 196-200, August 2013.

[8] S. Subramanian, M. Werner, S. Liu, J. Jose, R. Lupoaie, and X. Wu, "Congestion control for vehicular safety: synchronous and asynchronous mac algorithms," in Proceedings of the ninth ACM international workshop on Vehicular inter-networking, systems, and applications, pp. 63-72, ACM, June 2012.

[9] C. B. Math, A. Ozgur, S. H. de Groot, and H. Li, "Data rate based congestion control in V2V communication for traffic safety applications," in 2015 IEEE Symposium on Communications and Vehicular Technology in the Benelux (SCVT), pp. 1-6, November 2015.

[10] S. Yang, H. Kim, and S. Kuk, "Less is more: need to simplify ETSI distributed congestion control algorithm," Electronics Letters, vol. 50, pp. 279-281, February 2014.

[11] A. A. Gómez and C. F. Mecklenbräuker, "Dependability of decentralized congestion control for varying vanet density," IEEE Transactions on Vehicular Technology, vol. 65, pp. 9153-9167, January 2016.

[12] B. Cheng, A. Rostami, M. Gruteser, J. B. Kenney, G. Bansal, and K. Sjoberg, "Performance evaluation of a mixed vehicular network with CAM-DCC and LIMERIC vehicles," in 2015 IEEE 16th International Symposium on A World of Wireless, Mobile and Multimedia Networks (WoWMoM), pp. 1-6, IEEE, July 2015.

[13] A. Vinel, L. Lin, and N. Lyamin, "Vehicle-to-vehicle communication in C-ACC/platooning scenarios," IEEE Communications Magazine, vol. 53, pp. 192197, August 2015.

[14] N. Lyamin, A. Vinel, M. Jonsson, and B. Bellalta, "Cooperative awareness in VANETs: On ETSI EN 302 637-2 performance," IEEE Transactions on Vehicular Technology, vol. 67, pp. 17-28, January 2018.

[15] N. Lyamin, Q. Deng, and A. Vinel, "Study of the platooning fuel efficiency under ETSI ITS-G5 communications," in 2016 IEEE 19 th International Conference on Intelligent Transportation Systems (ITSC), pp. 551-556, November 2016. 